\title{
Intraocular Lens Fixation Technique Without Corneal Incision in Minimally Invasive Vitrectomized Eyes
}

\author{
Yimeng Sun $\cdot$ Zhaotian Zhang $\cdot$ Yantao Wei $\cdot$ Wei Chi
}

Shaochong Zhang

Received: October 25, 2021 / Accepted: January 12, 2022 / Published online: February 5, 2022

(C) The Author(s) 2022

\section{ABSTRACT}

Introduction: To introduce a modified technique for primary/secondary intraocular lens (IOL) fixation without corneal incision in vitrectomized eyes.

Methods: Consecutive case series who had undergone previous or concomitant pars plana vitrectomy (PPV) to have primary/secondary IOL fixation were prospectively included. A selfsealing scleral incision was made underneath the superior scleral flap, through which the IOL was inserted into the anterior chamber. The suture tied with the IOL passed through the sclera to fix the IOL in the ciliary sulcus.

Yimeng Sun and Zhaotian Zhang contributed equally to thearticle.

Supplementary Information The online version contains supplementary material available at https:// doi.org/10.1007/s40123-022-00464-4.

Y. Sun · Z. Zhang · Y. Wei · W. Chi

State Key Laboratory of Ophthalmology, Zhongshan

Ophthalmic Center, Sun Yat-sen University, Guangdong Provincial Key Laboratory of Ophthalmology and Visual Science, Guangdong Provincial Clinical Research Center for Ocular Diseases, Guangzhou 510060, China

S. Zhang ( $\square)$

Shenzhen Key Laboratory of Ophthalmology,

Shenzhen Eye Hospital Affiliated to Jinan

University, 18 Zetian Road, Shenzhen 518040,

Guangdong, China

e-mail: zhangshaochong@gzzoc.com
Patients were followed up for at least 3 months. Main outcomes were best corrected visual acuity (BCVA), intraocular pressure (IOP), surgically induced astigmatism (SIA), and intraoperative and postoperative complications.

Results: A total of 31 patients were included in the study. The mean follow-up time was $5.35 \pm 4.14$ months. The BCVA (log MAR unit) improved from $0.97 \pm 0.58$ preoperative to $0.42 \pm 0.36$ postoperative $(P<0.001)$. Mean IOP remained unchanged (preoperative IOP $14.03 \pm 2.90 \mathrm{mmHg}$, postoperative IOP $13.26 \pm 3.46 \mathrm{mmHg}, P=0.130$ ). The mean SIA was $0.91 \pm 0.76$ diopters. No obvious intraoperative and postoperative complications were observed.

Conclusion: This method has favorable postoperative visual recovery and IOP control. This modified method could be taken into account as an option by surgeons in vitreoretinal surgery.

Keywords: Intraocular lens; Surgical technique; Transscleral fixation; Vitrectomy 


\section{Key Summary Points}

Minimally invasive vitrectomy has been used extensively. Thus, surgical procedures of intraocular lens (IOL) fixation concomitant with vitrectomy need improvement.

We introduce a modified technique for primary/secondary IOL fixation with scleral tunnel incisions underneath the scleral flap instead of corneal incision.

Effectiveness and safety of this method combined with vitrectomy were first evaluated. The method is safe and efficacious with good intraocular pressure and anterior chamber control and visual recovery and low surgically induced astigmatism in vitrectomized eyes.

This novel, less-invasive IOL fixation technique could be an excellent option in the microincision vitrectomy era.

\section{DIGITAL FEATURES}

This article is published with digital features, including a video, to facilitate understanding of the article. To view digital features for this article, go to https://doi.org/10.6084/m9. figshare.18282557.

\section{INTRODUCTION}

Nowadays, microincision vitrectomy has become a standard approach to vitrectomy in the majority of circumstances and is characterized by its faster recovery and better visual outcomes [1]. Meanwhile a large number of intraocular lens (IOL) techniques have also transformed into a refractive surgery to pursue better vision restoration. Actually, loss of integrity of posterior capsule is the main circumstance encountered in vitrectomy; it compromise the following conditions:

complications of cataract surgery, crystalline lens subluxation or luxation such as Marfan syndrome, IOL dislocation, ocular trauma, etc. Therefore, IOL fixation is an important surgical technique to manage various eye conditions where there is aphakia without sufficient capsular support after vitrectomy. Use of minimally invasive IOL fixation techniques with high safety and efficiency during or after vitrectomy has posed management challenges for ophthalmologists.

Multiple surgical modalities can be utilized to fix IOL and each has its own advantages. The scleral-sutured IOL is a well-established, reliable method for IOL fixation which is mainly divided into sutureless scleral tunnel fixation and suture fixation. Recently, the sutureless intrascleral fixation technique has become popular. Yamane developed a double-needle technique and a flanged IOL fixation technique; these two techniques can provide good IOL fixation with firm haptic fixation without using sutures [2]. In the majority of IOL fixations, the corneal incision is created to externalize the suture and implant the IOL [3, 4], which leads to the use of more foldable IOLs and makes intraocular procedures easy to handle. Subsequently multiple innovative modifications based on the aforementioned method have been developed and widely used. There are issues that have been at the forefront of our thinking. As scarring of the cornea may cause postoperative refractive errors such as astigmatism, we want to reduce the operation on corneal tissue to obtain optimal optical quality and visual performance, especially for patients who have previously undergone phacoemulsification. Creation of a corneal incision may cause fluctuation of the intraocular pressure (IOP) and instability of the anterior chamber. Stability of the anterior chamber, especially in eyes that have undergone vitrectomy, is critical during IOL fixation surgery [5]. Herein, we describe a modified technique after vitrectomy for IOL fixation with scleral tunnel incisions underneath the scleral flap instead of corneal incision. 


\section{METHODS}

This is a prospective case series study. The study was performed in accordance with the regulations of the World Medical Association's Declaration of Helsinki. This study was approved by the institutional research committee of the Zhongshan Ophthalmic Center of Sun Yat-sen University (Guangzhou, China). All patients included in the study provided a written informed consent. All surgeries were performed by an experienced surgeon (S. Z.).

The patients who met the following inclusion criteria were prospectively recruited: Patients requiring primary/secondary fixation either combined with vitrectomy or in an eye that had previously undergone vitrectomy: aphakia, crystalline lens subluxation or luxation, and dislocated IOL. Patients whose postoperative follow-up time was less than 3 months as well as those who had other retinal diseases requiring treatment and thus were not fit for IOL fixation were excluded from the study. Patients with other serious eye conditions were also excluded.

Prior to the surgery, all patients underwent a comprehensive ophthalmologic examination that included measurement of BCVA using a decimal optometric chart, non-contact tonometry, slit-lamp examination, dilated fundoscopy, optical coherence tomography (Spectralis Heidelberg Engineering, Heidelberg, Germany), optical biometry (IOL Master, Carl Zeiss, Meditec, Dublin, CA), and Optometric screening by an optometrist in the Zhongshan Ophthalmic Center.

\section{Surgical Techniques and Procedures}

1. Expose sclera: A fornix-based conjunctival opening was then made inferiorly and superiorly and a blunt dissection was continued until the sclera was exposed.

2. Create scleral flaps: Partial-thick triangular scleral flaps were then created followed by light diathermy at the 12 and 6 o'clock meridians $2 \mathrm{~mm}$ posterior to the limbus.

3. Create a sclerotomy: A 10-0 polypropylene (10-0 Alcon Polypropylene Blue 12“ AUM-
5 Spatula and SC-5 Straight, Double Armed; Alcon laboratories, Fort Worth, TX, USA) with a curved needle and a straight needle at the end of the thread was then used to fix the IOL. The straight needle was used to create a sclerotomy $3 \mathrm{~mm}$ posterior to the limbus at 12 o'clock.

4. Place the suture for fixation: It was guided to the appropriate transscleral penetration

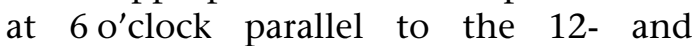
6-o'clock meridians and then withdrawn from the eye.

5. Place infusion: The inferotemporal infusion was left in position.

6. Create scleral incision: A 3.2-mm keratome (Sharpoint Surgical Specialties Corp, TX, USA) was then used to create a self-sealing incision through the superior scleral bed to the anterior chamber instead of a clear corneal incision.

7. Suture tied to the haptics: The 10-0 polypropylene was then draped out from the scleral incision and bisected. The two free ends of the 10-0 polypropylene thread were then tied to the IOL (Aspira-Aa, Humanoptics, Germany) haptics using regular 3-1-1 throws.

8. Insert IOL: The IOL was held and folded using forceps and then inserted into the posterior chamber through the scleral incision. It was adjusted into position to ensure that it was well centered.

9. Fix IOL in sclera: Two needles were then threaded through the sclera to form a snare that was tied to the suture to form a knot at the fixation point.

10. Close surgical incisions: The scleral flap was then closed using 8-0 polypropylene sutures and the knot buried under the scleral flap. The conjunctival opening was then finally closed using an 8-0 polypropylene sutures.

\section{Statistical Analysis}

The decimal BCVA was converted to the logarithm of the minimum angle of resolution ( $\log$ MAR). The surgically induced astigmatism 


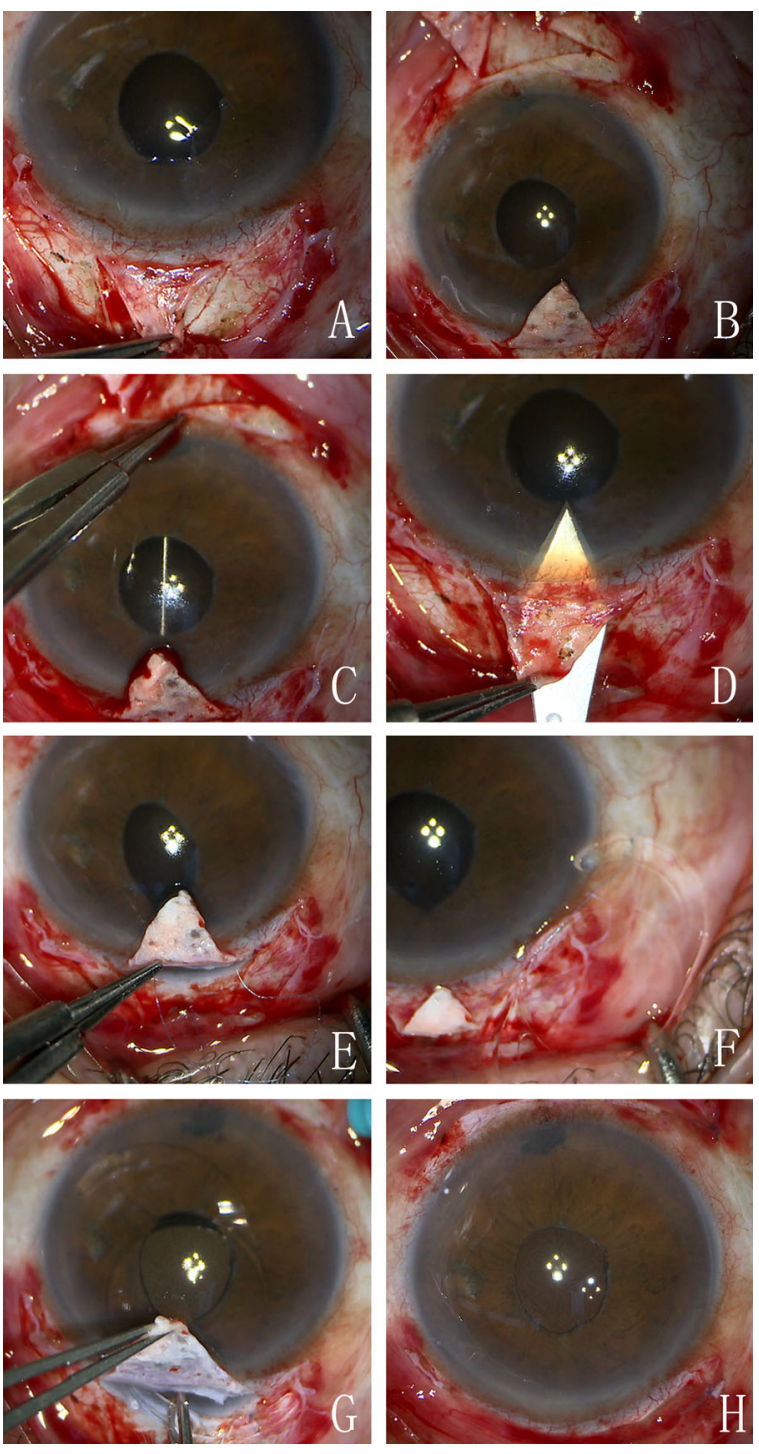

Fig. 1 Intraoperative view of the surgical procedures: a, b Partial-thick triangular scleral flaps were created at the 12 and 6 o'clock meridians $2 \mathrm{~mm}$ posterior to the limbus; the base of scleral flap at 12 o'clock is parallel to the corneal limbus (a). The base of scleral flap at 6 o'clock is perpendicular to the corneal limbus (b). c The straight needle was used to create a sclerotomy $3 \mathrm{~mm}$ posterior to the limbus and created an appropriate transscleral penetration parallel to the 12- and 6-o'clock meridians; d A self-sealing incision was made through the superior scleral bed to the anterior chamber; e The 10-0 polypropylene was draped out from the scleral incision and bisected. f The two free ends of the 10-0 polypropylene thread were then tied to the IOL. $g$ The IOL was inserted into the posterior chamber through the scleral incision; $\mathbf{h}$ The scleral flap and the conjunctival opening were closed
(SIA) was calculated using the formula SIA = $\sqrt{(B \cos 2 b-A \cos 2 a)^{2}+(B \sin 2 b-A \sin 2 a)^{2}}$ in accordance with Jaffe's vector analysis of SIA where $A$ and $a$ represent the power (diopters, D) and axis (degree) of preoperative astigmatism, respectively, and $B$ and $b$ represent the power (D) and axis (degree) of postoperative astigmatism, respectively [6]. Continuous data were in accordance with normal distribution and expressed as mean values \pm standard deviation. After normal distribution was established using quantile-quantile plots, BCVA and IOP (in line with normal distribution) before and after surgery 1 day, 1 month, and the longest time point of individual follow-up were collected and preoperative data and observation endpoint data were tested for significant differences using paired $t$ tests. Statistical analysis was done using SPSS software version 22.0 (SPSS Inc., Chicago, IL).

\section{RESULTS}

The study included 31 patients comprising 25 men and 6 women whose mean age was $48.06 \pm 17.53$ years. 12 patients underwent secondary surgery to correct aphakia after pars plana vitrectomy. Among the 12, 3 underwent phaco-vitrectomy because of traumatic injury, 4 underwent phaco-vitrectomy to treat retinal detachment, and 5 had dislocated lenses that were due for correction. The remaining 19 patients underwent primary surgery to correct dislocated IOL and crystalline lens subluxation or luxation. Patients' characteristics are summarized in Table 1.

The mean follow-up time was $5.35 \pm 4.14$ months. Their mean preoperative $\log$ MAR BCVA and postoperative log MAR BCVA at the final follow-up were $0.97 \pm 0.58$ and $0.42 \pm 0.36$, respectively $(P<0.001)$. There was no significant change in their mean IOP $(P=0.130)$. Their mean preoperative IOP was $14.03 \pm 2.90 \mathrm{mmHg}$ and the postoperative IOP was $13.26 \pm 3.46 \mathrm{mmHg}$. The SIA was $0.91 \pm 0.76 \mathrm{D}$ (Table 2). The IOL were well centered and no intraoperative and 
Table 1 General characteristics of patients and eyes undergoing scleral fixation of an intraocular lens combined with vitrectomy or in vitrectomized eyes

\begin{tabular}{ll}
\hline Age (years) & \\
Mean \pm SD & $48.06 \pm 17.53$ \\
Range & $7-65$ \\
Gender, no. (\%) & $25(80.65)$ \\
Male & $6(19.35)$ \\
Female & \\
Surgical indication, no. of eyes (\%) & $12(38.71)$ \\
Aphakia after phaco-vitrectomy & \\
(secondary) & 3 \\
Traumatic injury & 4 \\
Retinal detachment & 5 \\
Dislocated lenses & $9(29.03)$ \\
Crystalline lens subluxation or luxation & \\
(primary) & $17(32.26)$ \\
Dislocated IOL (primary) & \\
Interval before secondary surgery (months) \\
Mean \pm SD & 14 \\
Range & \\
PPV systems & \\
23 -gauge PPV & \\
\hline & \\
\hline &
\end{tabular}

$I O L$ intraocular lens, $P P V$ pars plana vitrectomy

postoperative complications were observed during the follow-up period.

\section{DISCUSSION}

IOL fixation is an important surgical technique for patients who need IOL implantation but have insufficient capsular support. Multiple surgical incisions to the anterior and posterior segment make it difficult to maintain the IOP and globe during the various maneuvers in the anterior and posterior segment. This leads to instability of the anterior chamber, thus making further surgical manipulations much harder [7]. In the current era of minimally invasive surgeries, vitrectomy has transitioned from large incisions to smaller incisions that have less inflammatory response and surgical trauma as well as faster postoperative recovery $[8,9]$. As such, the traditional IOL fixation technique is still challenging.

Malbran first reported transscleral sulcus fixation of the posterior chamber IOL in 1986 [10]. Ab externo two-point fixation is a frequently used surgical method. A straight needle was inserted $a b$ externo and retrieved at the opposite scleral bed. A loop of suture was hooked out of the eye from a corneal incision and cut. The ends of suture were tied to the haptics. The IOL was inserted into the eye also through the corneal incision [11]. In this study, a novel minimally invasive IOL fixation technique similar to the aforementioned was performed in vitrectomized eyes to correct varying eye conditions. Its safety and success rate were then further evaluated. Herein, the traditional sutured scleral IOL fixation technique was modified to conform to a minimally invasive surgery. A self-sealing scleral incision was created underneath the scleral flap instead of a corneal incision. The 10-0 polypropylene suture was externalized through the scleral incision made and the foldable IOL was also inserted through this incision and fixed in the ciliary sulcus.

This modified technique has several advantages. Firstly, it still retains the benefits of suture fixation of IOL which is widely used owing to its convenient adjustability of suture tension, IOL location, and stability of IOL haptics. Sutured fixation of the posterior chamber intraocular lens enables the IOL to be located closest to the original lens to get more optical benefits and furthest from the corneal endothelium and trabecular meshwork [12]. The straight needle and suture used in this technique are more favorable in creation of symmetrical intrascleral tunnels on either side of the limbus and avoid negative impact on the IOL tilt and optic 
Table 2 Comparison of ocular parameters before surgery and at the end of follow-up after surgery

\begin{tabular}{lllll}
\hline Characteristics & Data & & \\
\hline Follow-up time (months) & & & & \\
Mean \pm SD & $5.35 \pm 4.14$ & & & \\
Range & $3-24$ & & Postoperative 1 month & Observation endpoints \\
BCVA (log MAR) & Preoperative & Postoperative 1 day & $0.45 \pm 0.39$ & $0.42 \pm 0.36$ \\
Mean \pm SD & $0.97 \pm 0.58$ & $0.6 \pm 0.50$ & $0-1.3$ & $0-1.5$ \\
Range & $0.1-2$ & $0-1.8$ & Postoperative 1 month & Observation endpoints \\
IOP (mmHg) & Preoperative & Postoperative 1 day & $13.26 \pm 3.46$ \\
Mean \pm SD & $14.03 \pm 2.90$ & $11.90 \pm 2.70$ & $19 \pm 2.62$ & $8-20$ \\
Range & $8-19$ & $7-17$ & $10-19$ & \\
SIA (diopters), mean \pm SD & $0.91 \pm 0.76$ & & & \\
\hline
\end{tabular}

$B C V A$ best corrected visual acuity, $\log M A R$ logarithm of the minimum angle of resolution, IOP intraocular pressure, SIA surgically induced astigmatism, $S D$ standard deviation

centration [13]. It also has a universal applicability across different implantation types, suture types, and suturing techniques and requires no special instruments. Therefore, this method offers the much-needed postoperative stability and less instrument restriction while avoiding invasive and traumatic problems.

Secondly, the technique is less traumatic because of fewer manipulations on the cornea and well adapted to eyes in which minimally invasive vitrectomy was performed. The approach used in this study avoided the creation of a large corneal incision to reduce possible associated corneal astigmatism and allow rapid visual rehabilitation. This was particularly important to cataract surgery patients who had previously undergone vitrectomy due to posterior capsular rupture because they initially had a higher demand of postoperative vision recovery. The amount of SIA may range from $0.24 \mathrm{D}$ to $1.65 \mathrm{D}$ [14], depending on the size and location of the clear corneal incisions in cataract surgery with phacoemulsification [5]. And IOL tilt can also induce astigmatism and higherorder aberrations [15]. Herein, the mean SIA of $0.91 \mathrm{D}$ was comparable with the SIA mean reported after cataract surgery with incisions of similar sizes. Patients did not complain of halo or glare perception. In the same manner, the technique reduces corneal scar associated with ocular trauma, postsurgical astigmatism, and corneal endothelial injury, thus minimizing technical difficulties of subsequent surgical procedures on the cornea in trauma patients. As such, this technique can reduce refractive changes, protect cornea endothelial cells, and accelerate visual rehabilitation.

Third, the manipulations through corneal incision result in more distortion and distention of the incision, thus increasing the possibility of developing wound leaks. This characteristic is not favorable for the maintenance of the appropriate depth of the anterior chamber and IOP that may significantly increase the difficulty of subsequent surgeries as well as increase the risk of intraoperative complications even for eyes supported by the infusion cannula. Wound leaks after surgery can also cause hypotony, further leading to retinal detachment and choroidal effusion and increase the risk of endophthalmitis [16]. Herein, the incision created was beneath the scleral flap and at a safe distance away from the limbus. Pressure from the scleral flap also 
prevents incomplete closure of the incision, thus allowing stable maintenance of the IOP. Moreover, the incision did not expose to the outer environment. Instead, we hid the incision inside the sclera lamina, thus preventing pathogens from gaining access to the eye. The mean postoperative IOP was $13.26 \pm 3.46 \mathrm{mmHg}$ which is not significantly different from the preoperative values. Our patients indeed had a favorable postoperative IOP control with no intraoperative and postoperative complications. We also employed some measures to control IOP during and after surgery. We used infusion cannula from a trocar in the inferior-temporal quadrant to maintain intraoperative IOP. Czajka et al. found that hypotonia was more common in the trocar group compared with IOL fixation techniques without the use of a trocar [17]. Suren et al. showed that use of a smaller gauge trocar for PPV could significantly reduce postoperative hypotony. We used 23- or 25-gauge vitrectomy system and in these cases the paracentesis incision was sutured at the end of the surgery [18]. Nevertheless, this technique was limited by several factors. The incision size was limited by traditional IOL. Moreover, the technique was limited by the fact that conjunctival opening and exposure of sclera was still an unavoidable step in the surgery. In the most common scleral suture fixation techniques, the suture is tied on the scleral bed beneath a scleral flap or in a scleral groove or pocket. Scarring of conjunctiva and sclera is unavoidable and there are some studies about adjustable and less traumatic suturing technique for transscleral fixation, and future improvement can be expected [19].

\section{CONCLUSION}

The postoperative visual prognosis, refractive outcomes, and IOP control associated with a modified scleral incision IOL fixation technique were successfully evaluated. Indeed, this novel, less-invasive IOL fixation technique is an excellent option in the microincision vitrectomy era. However, a longer follow-up analysis is required to confirm the outcomes of the technique.

\section{ACKNOWLEDGEMENTS}

This study was approved by the institutional research committee of the Zhongshan Ophthalmic Center of Sun Yat-sen University (Guangzhou, China). We thank the participants of the study.

Funding. No funding or sponsorship was received for this study, the journal's Rapid Service Fees were funded by the authors.

Authorship. All named authors meet the International Committee of Medical Journal Editors (ICMJE) criteria for authorship for this article, take responsibility for the integrity of the work as a whole, and have given their approval for this version to be published.

Author Contributions. Yimeng Sun and Zhaotian Zhang contributed equally to the article. Yimeng Sun, Zhaotian Zhang and Shaochong Zhang contributed to the conception and design of the study. Shaochong Zhang performed the surgery; Yimeng Sun acquired the data and wrote the manuscript; Yimeng Sun and Zhaotian Zhang analyzed the data; Yantao Wei, Wei Chi and Shaochong Zhang supervised the project and reviewed the paper. All of the authors have read and approved the published version of the manuscript.

Disclosures. Yimeng Sun, Zhaotian Zhang, Yantao Wei, Wei Chi and Shaochong Zhang declare that the research was conducted in the absence of any commercial or financial relationships that could be construed as a potential conflict of interest.

Compliance with Ethics Guidelines. This is a prospective case series study performed in accordance with the regulations of the World Medical Association's Declaration of Helsinki. This study was approved by the institutional research committee of the Zhongshan Ophthalmic Center of Sun Yat-sen University (Guangzhou, China). 
Data Availability. The datasets used or analyzed during the current study are available from the corresponding author on reasonable request.

Open Access. This article is licensed under a Creative Commons Attribution-NonCommercial 4.0 International License, which permits any non-commercial use, sharing, adaptation, distribution and reproduction in any medium or format, as long as you give appropriate credit to the original author(s) and the source, provide a link to the Creative Commons licence, and indicate if changes were made. The images or other third party material in this article are included in the article's Creative Commons licence, unless indicated otherwise in a credit line to the material. If material is not included in the article's Creative Commons licence and your intended use is not permitted by statutory regulation or exceeds the permitted use, you will need to obtain permission directly from the copyright holder. To view a copy of this licence, visit http://creativecommons.org/licenses/bync/4.0/.

\section{REFERENCES}

1. Thompson JT. Advantages and limitations of small gauge vitrectomy. Surv Ophthalmol. 2011;56(2): 162-72. https://doi.org/10.1016/j.survophthal. 2010.08.003.

2. Yamane S, Sato S, Maruyama-Inoue M, Kadonosono K. Flanged intrascleral intraocular lens fixation with double-needle technique. Ophthalmology. 2017;124(8):1136-42. https://doi.org/10.1016/j. ophtha.2017.03.036.

3. Wagoner MD, Cox TA, Ariyasu RG, Jacobs DS, Karp CL. Intraocular lens implantation in the absence of capsular support: a report by the American Academy of Ophthalmology. Ophthalmology. 2003;110(4):840-59. https://doi.org/10.1016/ s0161-6420(02)02000-6.

4. Yoshida N, Kojima T, Yokoyama S, Horai R, Ichikawa K. New surgical approach for intrascleral fixation using an intraocular lens with hook-shaped haptics. J Cataract Refract Surg. 2018;44(2):129-33. https://doi.org/10.1016/j.jcrs.2017.12.011.
5. Solomon K, Gussler JR, Gussler C, Van Meter WS. Incidence and management of complications of transsclerally sutured posterior chamber lenses. J Cataract Refract Surg. 1993;19(4):488-93. https:// doi.org/10.1016/s0886-3350(13)80612-8.

6. Shah S, Chatterjee A, Doyle SJ, Bessant DA. Astigmatism induced by spherical photorefractive keratectomy corrections. Ophthalmology. 1997;104(8): 1317-20. https://doi.org/10.1016/s01616420(97)30141-9.

7. Ahn JK, Yu HG, Chung $\mathrm{H}$, Wee WR, Lee JH. Transscleral fixation of a foldable intraocular lens in aphakic vitrectomized eyes. J Cataract Refract Surg. 2003;29(12):2390-6. https://doi.org/10.1016/ s0886-3350(03)00338-9.

8. Recchia FM, Scott IU, Brown GC, Brown MM, Ho AC, Ip MS. Small-gauge pars plana vitrectomy: a report by the American Academy of Ophthalmology. Ophthalmology. 2010;117(9):1851-7. https:// doi.org/10.1016/j.ophtha.2010.06.014.

9. Todorich B, Thanos A, Woodward MA, Wolfe JD. Sutureless intrascleral fixation of secondary intraocular lens using 27-gauge vitrectomy system. Ophthalmic Surg Lasers Imaging Retina. 2016;47(4):376-9. https://doi.org/10.3928/ 23258160-20160324-14.

10. Malbran ES, Malbran E, Negri I. Lens guide suture for transport and fixation in secondary IOL implantation after intracapsular extraction. Int Ophthalmol. 1986;9(2-3):151-60. https://doi.org/ $10.1007 /$ BF00159844.

11. Lewis JS. Ab externo sulcus fixation. Ophthalmic Surg Lasers Imaging Retina. 1991;22(11):692-5.

12. Por YM, Lavin MJ. Techniques of intraocular lens suspension in the absence of capsular/zonular support. Surv Ophthalmol. 2005;50(5):429-62. https:// doi.org/10.1016/j.survophthal.2005.06.010.

13. Sugiura T, Kaji Y, Tanaka Y. Anatomy of the ciliary sulcus and the optimum site of needle passage for intraocular lens suture fixation in the living eye. J Cataract Refract Surg. 2018;44(10):1247-53. https://doi.org/10.1016/j.jcrs.2018.07.017.

14. Can I, Takmaz T, Yildiz Y, et al. Coaxial, microcoaxial, and biaxial microincision cataract surgery: prospective comparative study. J Cataract Refract Surg. 2010;36(5):740-6. https://doi.org/10.1016/j. jcrs.2009.11.013.

15. Choi SK, Kim JH, Lee D, Park SH, Maeda N, Ma KJ. IOL tilt and decentration. Ophthalmology. 2010;117(9):1862.e1-1862.e4. https://doi.org/10. 1016/j.ophtha.2010.03.078. 
16. Slade DS, Hater MA, Cionni RJ, Crandall AS. Ab externo scleral fixation of intraocular lens. J Cataract Refract Surg. 2012;38(8):1316-21. https:// doi.org/10.1016/j.jcrs.2012.05.022.

17. Czajka MP, Frajdenberg A, Stopa M, Pabin T, Johansson B, Jakobsson G. Sutureless intrascleral fixation using different three-piece posterior chamber intraocular lenses: a literature review of surgical techniques in cases of insufficient capsular support and a retrospective multicentre study. Acta Ophthalmol. 2020;98(3):224-36. https://doi.org/ 10.1111/aos.14307.
18. Suren E, Kalayci M, Cetinkaya E, et al. Evaluation of the findings of patients who underwent sutureless flanged transconjunctival intrascleral intraocular lens implantation with or without pars plana vitrectomy. J Ophthalmol. 2021;1(2021):4617583. https://doi.org/10.1155/2021/4617583.

19. Zhao P, Ou Z, Zhang Q, Jin H. Adjustable buckleslide suture: a novel surgical technique for transscleral fixation of intraocular lenses. Retina. 2019;39(Suppl 1):S24-9. https://doi.org/10.1097/ IAE.0000000000002207. 